\title{
Ni cinéma, ni télévision : la vidéo et les paradoxes de la représentation
}

Michèle Willems

\section{(2) OpenEdition \\ Journals}

\section{Édition électronique}

URL : http://journals.openedition.org/shakespeare/296

DOI : $10.4000 /$ shakespeare.296

ISSN : 2271-6424

\section{Éditeur}

Société Française Shakespeare

\section{Édition imprimée}

Date de publication : 1 novembre 1998

Pagination : 183-194

ISBN : 2-84269-230-6

\section{Référence électronique}

Michèle Willems, « Ni cinéma, ni télévision : la vidéo et les paradoxes de la représentation », Actes des congrès de la Société française Shakespeare [En ligne], 16 | 1998, mis en ligne le 01 novembre 2007, consulté le 03 mai 2019. URL : http://journals.openedition.org/shakespeare/296 ; DOI : 10.4000/ shakespeare.296 


\section{S H A K E S P E A R E \\ \& $\quad$ L E $\quad$ C I N É M A}

Société Française Shakespeare

Actes du Congrès de 1998

米 $*$ *

Textes réunis et présentés par

Patricia DORVAL

publiés sous la direction de

Jean-Marie MAGUIN 
Site web : <http : //alor.univ-montp3.fr/serinf/SFS/> Liste de diffusion : <sfs-1@smrl.univ-montp3.fr >

Tous droits de traduction, de reproduction et d'adaptation réservés pour tous les pays.

(C) 1998. Société Française Shakespeare,

École Normale Supérieure, 45 rue d'Ulm. 75005 Paris.

ISBN 2-84269-230-6 


\section{N I C I N É M A, N I T ÉLÉ V IS I O N : L A VIDÉO ET LES PARADOXES DE LA R E P R É S E T A T I O N}

Le spectateur qui, en quittant l'un des théâtres de Stratford, souhaite prolonger le plaisir d'une représentation de Hamlet par exemple, se voit offrir, dans les boutiques de la RSC, un vaste choix de vidéocassettes. Les films y figurent en bonne place, depuis celui de Laurence Olivier ou de Zeffirelli jusqu'à la dernière réalisation de Branagh. Sur le même rayon, il trouvera aussi des copies de théâtre filmé, comme la mise en scène de Tony Richardson (avec Nicol Williamson) enregistrée à Londres en 1969 et commercialisée en 1991, ou celle de John Gielgud (avec Richard Burton), filmée à New York en 1964 et restaurće en $1995^{2}$. Paradoxalement, le télefilm de la BBC n'étant pas actuellement commercialisé, il n'aura guère l'occasion d'acquérir une version de la pièce effectivement prévue pour son petit écran, mais il pourra se consoler en regardant le dessin animé proposé dans la série des Animated Tales, avec la caution scientifique de Stanley Wells ${ }^{3}$.

Ce qu'on a appelé «l'industrie Shakespeare» se porte bien car, depuis ses premières manifestations lors du Jubilé organisé par Garrick à Stratford en 1769 , ellc a su s'adapter et se diversifier. Le temps n'est plus où l'horloger Thomas Sharp, qui avait eu la prévoyance d'acquérir le mûrier abattu dans le jardin de New Place, inondait le marché du souvenir shakespearien d'une telle quantité de reliques qu'il se trouva des esprits chagrins pour s'interroger sur la taille de cet arbre mythique. De nos jours, le créneau porteur est plutôt le marché de l'éducation, qui surfe désormais sur la vague des technologies nouvelles et du multi-média et qui toujours s'appuie sur l'enregistrement vidéo. Dans les années quatre-vingts déjà, la production du Shakespeare 
Complet télévisé pour la BBC fut largement motivée par la possibilité de commercialiser la série dans le monde entier, grâce à la vidéo, auprès de populations universitaires frustrées de théâtre mais non de crédits. La mise en conserve du canon shakespearien (le $T L S$ du 10 mai 1986 salua ironiquement le bouclage du projet en titrant "The canon in the can») justifiait l'investissement de divers capitaux, en particulier américains. Et depuis une bonne décennie, les vidéocassettes de tous types se multiplient: le film shakespearien, comme les autres films, fait une deuxième carrière sur nos petits écrans; les mises en scène de théâtre, filmées in situ, en studio ou seulement pour archivage, se donnent l'illusion de jouir d'une vie éternelle ; et il faut ajouter à ces enregistrements divers (dont certains sont piratés), les technologies nouvelles qui utilisent les vidéo-clips, comme les CD-Roms (qui accompagnent même les éditions savantes) ${ }^{4}$, les programmes interactif's qui nous font pénétrer dans le monde virtuel de Shakespeare ${ }^{5} \mathrm{ct}$, bien sûr, Internet; notre collègue américain Michael Mullin rassemble, pour son «Shakespeare Website», des vidéo-clips de toutes sortes, y compris.des enregistrements de représentations shakespeariennes qu'il glane lui-même, caméscope au poing, dans le monde entier.

C'est dire si l'étiquette «vidéo» est une catégorie vague et élastique, qui risque, si l'on n'y prend pas garde, d'être facteur de confusion, au plan théorique comme au plan pédagogique. Mes étudiants de Licence, qui ont Macbeth à leur programme, ont la possibilité de visionner, outre le film d'Orson Welles, celui de Polanski, le téléfilm de la BBC, ou encore la célèbre mise en scène de Trevor Nunn pour The Other Place, filmée en studio pour la télévision. Leur premier réflexe est souvent de venir voir «unc vidéo de Macbeth», quand ce n'est pas «la vidéo de Macbeth». Pour ajouter à la confusion, la Faculté vient de nous équiper d'un système de vidéo-projection sur grand écran : alors que nous étions habitués à déchiffrer les travellings et les panoramiques de Polanski sur un écran de télévision, il a fallu reculer de trois rangs pour réussir à lire les gros-plans de Gold ou de Trevor Nunn, soudain frappés de gigantisme sur grand écran. Le téléfilm avait soudain acquis les dimensions du film ${ }^{6}$, inversant du même coup les réflexes des drogués du magnétoscope qui désertent les salles obscures pour regarder, entre deux bandes noires, les films en cinémascope dans leur salon.

L'objet de ces longs préliminaires était d'établir le statut hybride de la vidéo, d'ailleurs souvent issue de croisements (entre théâtre et télévision par exemple), et donc sa propension à brouiller les cartes théoriques ct pédagogiques pour qui ne prend pas conscience que la donne a changé. En effet, film, téléfim et mise en scène filmée ne deviennent pas des transcodages équivalents de la pièce de théâtre originelle sous prétexte qu'on les visionne tous sur le même écran ${ }^{7}$. En fait, la vidéo peut rarement être considérée comme un média à part entière. La série des Animated Tales constitue 
l'exception puisque le média d'origine et son mode de diffusion se confondent. Mais la vidéo n'est assimilable ni au cinéma, ni à la télévision; au service des deux, ct du théâtre en sus, elle risque d'opérer un nivellement entre ces divers médias en occultant la spécificité de leurs codes. Et d'autres paramètres essentiels se trouvent déplacés: les conditions de réception et le statut du récepteur sont modifiés dès lors que la représentation est détournée de ses visées initiales par l'intervention de la vidéo et que film, téléfilm et même mise en scène de théâtre deviennent des produits commerciaux, comme l'indiquent ies notices figurant sur les boites: l'Othello de Parker y est décrit comme «a classic tale of love, passion, jealousy and betrayal» et le Richard III de Loncraine est ainsi résumé : «kidnap, seduction, betrayal and bloody murder» ${ }^{8}$. Il n'empêche que, moyennant certaines mises en garde, ces ressources nouvelles peuvent enrichir considérablement la réflexion sur la représentation du théâtre shakespearien ; c'est pourquoi il m'a paru utile d'analyser les effets, souvent paradoxaux, de la propagation de cet outil de travail, dont on n'étudie pas souvent les spécificités.

On a assez souvent, en revanche, comparé les sémiotiques du théâtre et du cinéma d'une part, du théâtre et de la télévision d'autre part et souligné que chaque média était régi par ses propres codes, pour que l'on puisse se contenter ici de quelques rappels, à partir de l'étude comparée de trois extraits, de film, de téléfilm et de théâtre filmé, qui devront malheureusement, pour des raisons de temps, rester extrêmement courts. Il s'agit de l'annonce faite à Macheth (1. 3), après le départ des sorcières, qu'il a été nommé «Thane of Cawdor». L'intérêt de cette fin de scène est qu'elle alterne dialogue, aparté et monologue. On examinera la même séquence, d'abord dans le film de Polanski (1971), puis dans la mise en scène de Trevor Nunn (1976), filmée en 1978 dans un studio de Thames Television après avoir été originellement calibréc pour la scène du théâtre de poche de Stratford et enfin dans le téléfilm BBC réalisé par Jack Gold (1982). Il ne s'agit, ni d'analyser les interprétations ou le jeu des acteurs, ni de hiérarchiser les diverses versions proposées mais d'apprécier que, bien que le mode de diffusion commun soit la vidéo et le cadre le petit écran, les stratégies de transcodage qui participent à la mise en image d'une pièce de théâtre peuvent être très différentes.

Le passage de la scène au grand écran, c'est à dire d'un art fondé sur la parole à un art fondé sur l'image, implique, c'est un lieu commun, un changement de relation entre le verbal et le visuel, que Kosintzev définit plus précisément comme «shifting the stress from the aural to the visual» ${ }^{9}$. La réponse de Polanski à cet impératif est claire : sa stratégie spatiale privilégie les extérieurs et utilise les déplacements, la gestuelle, les accessoires et les mouvements de caméra plus que le discours, pour impliquer le spectateur dans une histoire. Son film ne peut certes se vanter, comme une vidéo concurrente, d'être «authentically set in eleventh-century Scotland» ${ }^{10} \ldots$, puisqu'il fut 
tourné au pays de Galles; mais le paysage où deux soldats se déplacent à cheval en échangeant des paroles amplifiées par l'écho est pittoresque et désert à souhait. L'écran, comme le quatrième mur du théâtre naturaliste, ouvre sur le monde de l'illusion totale. La brève méditation, en voix off, de Macbeth allongé sous sa tente (sur quatre vers déplacés de la réponse qu'il avait faite aux sorcières) suggère le passage du temps. Son lever est filmé en temps récl, avec gestes réalistes à l'appui mais sans aucune parole. Le texte est très raccourci mais étiré dans le temps et dans l'espace, souvent aussi déconnecté de l'image, comme lorsque les mises en garde de Banquo sur «the instruments of darkness" s'accompagnent d'un gros plan sur les sandales que Macbeth est en train de lacer ", ou que ses préparatifs de départ (il prend son épée, se jette une couverture sur les épaules) ponctuent sa méditation. La voix ofl est utilisée pour l'aparté puis pour le monologue, seulement interrompu par la proclamation à voix haute de «I am Thane of Cawdor», soulignée par un gros plan plus signifiant sur la chaîne et la médaille, insignes de son nouveau titre. Le public est en situation d'espion privilégié, mais pas de partenaire car il n'y a jamais de rapport frontal.

Les choses se passent très différemment, et dans la mise en scène filméc et dans le téléfilm, en partie parce que le petit écran ne dispose ni du nême espace que le film ni de ses ressources pour le meubler; les mouvements de caméra se limitent au champ-contrechamp auquel le film recourt lui aussi pour les dialogues, mais les plans généraux sont remplacés par un cadrage sur les personnages, souvent même exclusivement sur leurs têtes. Couvert alternativement par quatre caméras, l'espace que Nunn a initialement visualisé comme un cercle ritucl où chaque acteur pénètre à son tour, reste nu, indéterminé, quasiment confondu avec celui qu'occupent les personnages, filmés sur fond noir et en gros-plans qui deviennent vite oppressants. L'absence de profondeur de champ et le recours au flou, ajoutés à la cohabitation des soldats en armure avec des messagers qui ressemblent à des diplomates contemporains, situent la représentation hors du temps. Dans cet univers claustrophobique et surréel, la densité du texte éclate, sans pauses ni silences, sans bruitage ni appoint musical. Pour reprendre la distinction établie par Christian Metz ${ }^{12}$, la parole, sujette au cinéma, redevient, comme au théâtre, souveraine. Dans les deux représentations, les monologues s'adressent directement à la caméra ; Ian McKellen joue sur les conventions théâtrales en lançant l'aparté par dessus son épaule, alors que le téléfilm utilise la voix off. Comme pour son décor de lande à petit budget, Gold recourt ici au langage cinématographique et aux options «réalistes» que l'on associe traditionnellement avec le petit écran. Mais ce dernier offre un support verbal et auditif plus important que le grand écran. Le texte, sans aucune coupure, est souvent prononcé, comme aussi dans la mise en scène filmée, face à la caméra ; il instaure, en particulier lors du monologue, un rapport de 
plus en plus intense entre le téléspectateur et le protagoniste. Le gros plan sur Ian McKellen intègre ses mains que l'on voit trembler. Le cadrage sur Nicol Williamson se resserre à mesure que s'installe la voix rauque du personnage habité par le mal, dont le regard semble aussi devenir intérieur, en particulier lorsqu'il prononce le distique. Le contraste entre la voix publique et la voix privéc apparaît pour la première fois au moment où, la caméra ayant tourné lentement pour reprendre les trois autres personnages dans le champ, Macbeth les rejoint avec quelques paroles équivoques. Ce type de pédagogie du texte n'est guère opéré par la caméra de cinéma qui dilue plutôt le texte dans l'espace. De la même manière, elle n'utilise guère les plans de coupe pour souligner la réaction d'un personnage à des paroles prononcées par un autre (ce qu'on appelle plus brièvement en anglais «a reaction shot») : dans toutes les représentations, la caméra traque la réaction de Macheth (et parfois de Banquo) à la nomination, mais celle de Nunn saisit aussi un regard complice de Ian McKellen lorsqu'Angus rappelle la traîtrise de Cawdor, et celle de Gold ne quitte plus Williamson jusqu'à l'aparté en voix off. Le public, complice et partenaire, accompagne la caméra dans son exploration du texte.

Le statut du texte est, on le voit, l'une des variantes essentielles dans les divers transcodages subis par la pièce de théâtre ${ }^{13}$. Mais ce statut peut aussi se trouver perturbé par l'intervention de la vidéo qui a décidément pour effet de brouiller les conventions de représentation spécifiques à chaque média. Ainsi, j'avais noté en $1987^{14}$, que la série BBC poursuivait des objectifs contradictoires en ciblant à la fois un public universitaire (auquel elle ambitionnait de fournir une «Intégrale» du texte shakespearien sous forme de vidéothèque avec tous les choix de "fidélité» et d'académisme que cela impliquait) et le téléspectateur, qui se serait sans doute satisfait de mises en images plus nerveuses et plus audacieuses. 11 m'apparaît plus clairement aujourd'hui que l'échec relatif de cet ambitieux projet est en partie imputable à cette tension non résolue entre vidéo et télévision lesquelles utilisent, certes, le même écran, mais ne visaient pas, en l'occurrence, le même public. D'autant que le problème à résoudre n'ayant été ni explicité ni soumis à discussion, chaque réalisateur tenta d'y apporter ses propres réponses, qui allèrent du recours au naturalisme cinématographique, censé répondre aux attentes du télespectateur qui découvrait Shakespeare, à l'esthétisme pictorialiste dont les citations visuelles s'adressaient plutôt à un public averti. La scule consigne, d'ailleurs diversement suivie, concernait la fidélité au texte ; ce qui explique que l'extrait du téléfilm de Gold n'ait subi aucune coupure, contrairement même à celui de la mise en scène filmée.

Parce qu'elle détermine bien des options et souvent des transpositions, la nature du public de chaque média constitue un des paramètres de l'analyse. Imaginerait-on d'étudier.le Henry $V$ d'Olivier en oubliant que le film fut dédié aux parachutistes qui débarquèrent en Normandie en 1944 ? Ou, pour passer à 
l'autre extrême, peut-on analyser les Animated Tales ${ }^{15}$ sans prendre en compte les ingrédients obligés de la culture dite «jeune» (dessin animé, BD, vidéo-clips, texte réduit à quelques bulles), qui définit globalement le public visé par le produit, c'est à dire l'adolescent moyen contraint par le système scolaire de s'intéresser à une pièce de Shakespeare, alors qu'on le crédite d'une capacité de concentration d'une demi-heure ${ }^{16}$ ?

Mais les facteurs d'appréciation sont d'autant plus complexes lorsque la représentation, déjà soumise à un transcodage, est détournée de son public et de son média de départ. Il serait intéressant de savoir si les réalisatcurs de films à grand public et à grand spectacle gardent à l'esprit, quand ils font lcurs choix cinématographiques, la vidéo qui, six mois après la première distribution du film, permettra à quelques personnes dans un salon de prendre le relais du vaste public rassemblé dans une salle obscure. A-t-on pensé à mon petit écran lorsqu'on a coulé le Titanic ou fait exploser le fantôme du vicil Hamlet en une séric d'effets spéciaux ? En revanche peut-être Branagh espèret-il que le texte shakespearien, dont il a obtenu de préserver la quasiintégralité, co-habitera mieux avec son spectacle hollywoodien sur le petit que sur le grand écran. Mais de telles distinctions sont en train de se périmer. Des perfectionnements techniques comme le laser-vidéo, le système Dolby Pro Logic et l'image numérique, ajoutés à la multiplication des téléviscurs 16/9, feront bientôt disparaître les différences entre la salle obscure et le cinćma à domicile, déjà cher aux Américains. A l'inverse, projetez une vidéo de téléfilm à un parterre d'étudiants, dans une salle de classe, et vous recrée $z$ un public de cinéma, avec ses réactions spontanées, et en particulier la contagion du rire qui modifie complètement le rapport à la comédie ${ }^{17}$. Les frontières entre les média sont en perpétuelle mutation.

La mutation essentielle, à mon sens, qui n'a pas fini de bouleverser nos approches, nos catégories et même notre perception du texte, est celle qui affecte la représentation théâtrale. On la met en conserve sous des formes diverses, on l'archive ${ }^{18}$; on peut même, on l'a vu, restaurer le film d'une représentation d'il y a trente ans ${ }^{19}$. La vidéo, dit la publicité, est la mémoire du cinéma. Serait-clle aussi celle du théâtre ? Le peut-elle ? Souvenons-nous quand même que dès lors que le spectacle est filmé, l'écran, comme l'explique André Bazin ${ }^{20}$, constilue un cache qui ne laisse voir au spectateur que ce que le réalisateur a choisi de lui montrer. Quelles que soient les conditions de l'enregistrement ou le nombre de caméras utilisées, le cadrage est toujours imposé ; le regard du spectateur n'a aucune chance de s'égarer sur le hors champ. Le théâtre à l'écran exclut la perception simultanée, celle qui, par exemple, permet d'apprécier la pièce dans la pièce, si difficile à transcrire à l'écran. Même filmée dans un studio semblable au lieu théâtral d'origine, la mise en scène de Nunn est soumise à une médiation supplémentaire : la mise en images. On peut certes se dire, comme dans le cas des reportages sportifs à 
la télévision, que la caméra sélectionne l'essentiel, qu'elle est comme on dit «sur la balle», voire que le plan et en particulier le gros-plan isolent ce qu'il faut voir ou savoir. Mais de ce fait, la réorganisation des signes qui s'opère au théâtre est considérablement limitée. La représentation est donnée, son sens est scellé.

Avec la vidéo de théâtre, nous sommes au coeur du paradoxe, puisque le spectacle, par essence mouvant et éphémère, se trouve figé, immobilisé, fixé pour des reproductions multiples; la cérémonic, c'est un trait de notre civilisation du caméscope, perd son unicité, son immédiateté ; elle se banalise. À la fois présente et absente, elle relève d'une virtualité sans cesse consultable. Et il faut ici prendre en compte les possibilités d'intervention offertes par le magnétoscope, qui viennent révolutionner le statut du spectateur. Légendairement décrit comme passif au cinéma, voyeur à la tćlévision, réceptif et vigilant au théâtre, le voilà soudain investi, par la grâce d'une télécommande, du pouvoir d'interrompre le spectacle, de l'accélérer, de revenir en arrière, de s'arrêter sur l'image, bref de contrôler, voire de dépecer la représentation.

Il n'est pas besoin d'insister sur les avantages ainsi procurés au chercheur qui peut analyser une mise en scène avec une précision et une sûreté que plusieurs visites au théâtre ne lui permettraient pas d'égaler. La représentation enregistrée constitue comme un texte-bis : avec ses re-lectures possibles, scs repérages de motifs visuels, sa progression plus ou moins rapide ou ses pauses pour méditer sur une difficulté, l'analyse critique d'une scène à l'écran est semblable à celle de la même scène sur la page. La comparaison des deux peut aussi faire saisir que toute représentation s'inscrit dans les blancs du texte, mais qu'elle est aussi fondée sur des choix (de mise en scìne, de décor, d'acteur, de jeu..) qui en excluent d'autres.

La réflexion sur les effets de la vidéo semble donc se structurer autour d'une dialectique de la rigidité et de la souplesse, particulièrement utile au plan pédagogique. Shakespeare sur vidéocassettes est certes un produit congelé, un crsatz de représentation, mais consultable à l'infini, en entier ou par morceaux. Ainsi chargé de permanence, il peut s'investir d'une autorité que n'avaient, à l'origine, ni le film, ni le téléfilm, ni la représentation filmée ; mais en même temps que la vidéo fait accéder le Macbeth d'Orson Welles au statut de classique, ou donne à la mise en scène de Nunn un caractère exemplaire, elle les transforme aussi en documents qui datent et en quelques représentations parmi d'autres. La possibilité de visionner de nombreux enregistrements permet en effet de conjurer le risque de verrouiller les interprétations; elle fait apparaître qu'une représentation donnée n'est ni la vidéo, ni une vidéo de Macbeth ${ }_{2}$ mais le résultat d'une conjonction de paramètres et de conditions singulières dont il faut tenir compte. En fait, la vidéo désacralise autant qu'elle canonise. Ainsi, il est fort probable que la 
dissémination du texte shakespearien sous des formes diverses par voic de vidéo, a partici é à la déstabilisation du «Shakespeare Canon» qui a marqué les dernières cúcennics. De transcodage en transposition en adaptation, de film en téléfilm en mise en scène filmée, la vidéo rend quasiment tangible la pluralité textuelle, à travers l'éternelle réécriture à laquelle la représentation soumet la pièce d'origine : il n'y a pas de lecture unique : si l'on exclut quelques lectures impossibles liées à la primauté de la matrice théâtrale ${ }^{21}$, on découvre l'infïnité des possibles qui affleurent selon les publics et les conditions de production.

La multiplication des vidéocassettes qui rendent accessibles une variété de représentations shakespeariennes ouvre désormais de vastes champs à l'enseignement et à la recherche : comparaisons entre les divers types de transcodages auxquels les différents médias soumettent le texte shakespearien ; approche du texte comme une partition que chaque metteur en scène interprètc selon ses options de départ; étude des choix de transposition en relation avec les attentes d'un public donné et, à mesure que vidéothèques et archives se meltent en place, reconstitution de l'histoire théâtrale d'une pièce avec prise en compte de l'historicité de chaque représentation. La liste est loin d'être close, car la vidéo participe au développement d'une culture médiatique shakespearienne qui encourage l'interactivité et l'interpicturalité entre les médias et conditionne, même au théâtre, les attentes du public et de la critique ${ }^{22}$. D'autant que la représentation dite "post-moderne», avec son système d'auto-références, de citations visuelles, de renvois et de clins d'(xil fonctionne de plus en plus comme une re-présentation.

Mais la vidéo shakespearienne, on l'a vu, est un matériau hybride qui a une fâcheuse tendance à bouleverser les catégories traditionnelles, à nous glisser entre les doigts. Il importe donc avant tout de n'aborder ce Caliban de nos médias qu'avec la prudence et la circonspection d'un Trinculo, en commençant, comme lui, par nous poser la question essentielle : "What have we here, a bian or a fish?"».

Michèle WILLEMS

Centre d'Études du Théâtre Anglo-saxon

Université de Rouen 


\section{N O T E S}

1 Une version remaniée de cet article sera publiée, en anglais, dans The Cambridge Companion to Shakespeare on Film, à paraître en 1999.

2 Joué au «Lunt-Fontanne Theatre», à New York, il fut projeté deux soirs de suite, simultanément dans divers thêâtres américains.

${ }^{3}$ La série, dont le titre rappelle l'entreprise de Charles Lamb, commercialisa d'ahord six pièces (Macbeth, Romeo and Juliet, A Midsummer Night's Dream, Tw'elfth Night, The Tempest et Hamlet) puis, devant le succès rencontré, en traita six autres (Julius Caesar, Richard III. The Winter's Tale, The Taming of the Shrew et (Othello). Leon Garfield fut chargé d'abréger le texte, ce qu'il fit en introduisant un narrateur et en maintenant des bribes de dialogue. Les dessins animés furent faits a Moscou et les textes dits par des acteurs de la RSC ou du National Theatre. La séric, qui fournit une livret éducatif avec la vidéo, a été traduite en 37 langues.

${ }^{4}$ Ainsi le Norton Shakespeare de Stephen Greenblatt, fut lancé au printemps dernier accompagné d'un CD-Rom de démonstration, pour l'instant limité à $A$ Midsummer Night's Dream mais annonçant le Norton Shakespeare Workshop pour six pièces.

${ }^{5}$ Le catalogue multimedia de Heffers (automne 1997) annonce «The Interactive Guide» d'Othello, qui s'appuie entre autres sur des extraits de cinq versions filmées.

${ }^{6}$ En fait, la vidéo, même projetée sur grand écran, ne reproduit pas véritahlement l'effet du film qu'il faudrait projeter dans sa version $35 \mathrm{~mm}$.

${ }^{7}$ L'irremplaçable Shakespeare on Screen, An International Filmography and Videography de K.S. Rothwell \& A.H. Melzer, London, 1990, propose des distinctions fort utiles: video / teleplay pour le Macbeth de Gold, video / interpretation pour celui de Nunn, à ne pas confondre avec motion picture / adaptation pour le film de Polanski. Au-delà de ces classifications, la critique commence à s'intéresser aux effets spécifiques de la vidéo, puisque la collection d'essais récemment publiés chez Routlege par L.E. Boose et $\mathrm{R}$. Burt (London \& New York, 1997), sous le titre Shakespeare, The Movie, précise dans son souslitre: Popularising the Plays on Film, Television and Video et prend effectivement en compte les retombées de la «culture video».

${ }^{8}$ Les textes qui apparaissent au dos des vidéocassettes sont toujours révélateurs. Le Richard $I I I$ de Loncraine poursuit ainsi sa publicité: "The hloodbath [Richard] unleashes takes its terrible toll on family and friends...», scelléc par la mise en garde finale : «suitable only for persons of 15 years and over", pudeur que le théâtre n'est pas contraint d'avoir. De la même manière, le texte de lancement du Hamlet de Branagh joue à la fois sur le sensationalisme et sur la réduction du politique, voire du cosmique, au familial (la vidéo étant destinée à «private domestic viewing»): «Hamlet (Branagh), the Prince of Denmark, is thrown into despair when his recently widowed mother, Gertrude (Julie Christie) marries his father's brother Claudius (Derek Jacobi). A terrifying confrontation with his father's ghost reveals to Hamlet that his father was murdered by Claudius, 
who, by his actions won both the throne of Denmark and Hamlet's mother». La vidéo, en grossissant les traits du cinéma commercial, les rend plus perceptibles.

${ }^{9}$ Cité par Jorgens dans Shakespeare on Film, p. 10.

10 II s'agit d'un Macbeth («winner of the 30th US film and video festival») réalisé par un certain Jeremy Freeston, avec Connery, dont on découvre qu'il se prénomme Jason, dans le rôle-titre.

"L'extrait sélectionné est loin de présenter un exemple extrême de priorité donnée au visuel et de libertés prises avec le texte, qui peuvent déboucher sur des adaptations souvent doublées de transpositions. Voir le Richard III de Loncraine, avec Ian McKellen, Ie Romeo + Juliet de Balz Luhrman ou le Twelfth Night de Trevor Nunn. La catćgoric «film shakespearien» recouvre d'ailleurs elle aussi des réalités très diverses qui vont de Throne of Blood, où Kurosawa exploite les conventions du Nô et du film de Samuraï pour rendre l'esprit, mais pas la lettre. de Macbeth, jusqu'au Hamlet de Branagh, plus intégral que la plupart des versions scéniques, en passant par toutes les variétés d'adaptations et de transpositions.

12 Voir Essais sur la signification au cinéma, 2 vol., Paris. Klincksieck. 1972, vol. I, p. 60.

13 Dans l'interview qu'il a accordée à François Laroque, Trevor Nunn rappelle la discipline qu'il s'est toujours imposée pour la télévision: «shooting the text rather than shooting the action», Cahiers élisabéthains, $n^{\circ} 52$, oct. 1997, p. 89.

14 Dans mon article «Verbal-Visual, Verbal-Pictorial or Textual-Televisual? Reflections on the BBC Shakespeare Series», publie dans Shakespeare Survey, n³9, 1989, p. 91-102, ensuite réimprimé dans Shakespeare and the Moving Image (A. Davies \& S. Wells, eds), Cambridge University Press, 1994, p. 69-85.

15 Voir à ce sujet l'essai de Laurie E. Osborne, «Poetry in Motion : Animating Shakespeare» dans Shakespeare, The Movie, p. 103-18.

${ }^{16}$ On pourrait affiner l'étude en introduisant un autre paramètre lić aux conditions de production : la tension entre la Grande-Bretagne et les États-Unis, dès lors que l'hégémonic culturelle américaine tente de s'exercer sur le Barde national ; cela explique, en l'occurrence, que ce projet gallois salué par le Prince Charles, ait opté pour un dessin animé soviétique plutôt que pour les conventions héritées de Walt Disney. Plus largement, la concurrence qui s'cxerce entre les deux pays autour d'un atout culturel prestigieux a pris un tour nouveau depuis que le dramaturge est considéré comme un enjeu cinématographique et commercial. Ces diverses données apparaissaient clairement dans le film d'Al Pacino, Looking for Richard, avec son apparent complexe à l'égard de Shakespeare, son montage désinvolte des interviews d'universitaires britanniques et même l'intervention de John Gielgud qui résumait, de manière quasi-caricaturale, le sentiment de supériorité des Britanniques sur le sujet de Shakespeare. En pratiquant non pas le théâtre filmé mais le théâtre dans le film, Al Pacino innove et «américanise» en quelque sorte l'approche théâtrale souvent considérée comme plus spécifiquement britannique. Ces mêmes paramètres pourraient sans doute fournir une explication parmi d'autres à la coexistence parfois difficile, dans les films de plus en plus américanisés de Branagh, entre des choix textuels qui correspondent à une approche théâtrale et culturelle «britannique» et des options cinématographiques 
et commerciales qui relèvent plutôt du grand spectacle et du «star-system» hollywoodiens.

17 Certains se souviendront de la surprise et de la satisfaction de Jack Gold, aussi réalisateur du Marchand de Venise, lorsque la scène entre les deux Gobbo, projetéc devant les participants au congrès de Rouen (en 1985), fut accueillie par des rires qu'il n'espérait pas devant un écran de télévision. Voir Shakespeare à la télévision, éd. Michèle Willems, Publications de l'Université de Rouen, 1987. p. 65 .

${ }^{18}$ Le marché propose beaucoup de vidéos de théâtre filmé, avec ou sans public, par une caméra unique qui restitue «le point de vue du monsieur de l'orchestre», comme l'écrit André Bazin (voir le chapitre "Théâtre et cinéma» dans son ouvrage Qu'est-ce que le cinéma?, Paris, 1958, p. 130-78). Ce type d'enregistrement est utilisé par la RSC et le Shakespeare Festival de Stratford, Ontario pour leurs archives (accessibles aux chercheurs), mais sert aussi à téléviser, puis à commercialiser sur vidéocassettes, des mises en scène qui ont rencontré un certain succès à la scène. L'un des premiers excmples en est le Hamlet de Tony Richardson, avec Nicol Williamson, filmé en 1969 à la Round House, à Londres. Le même type d'enregistrement de théâtre vivant fut pratiqué pour la série des drames historiques, The Wars of the Roses, mis en scène par Michael Bogdanov pour l'English Shakespeare Company. (The Wars of the Roses, mis en scène en 1964 par John Barton pour la RSC et filmé sur scène pour la BBC antéricurement à l'avènement de la vidéo, ne subsiste que dans les archives de la RSC.). Plus récemment, le Richard II de Deborah Warner avec Fiona Shaw dans le rôle titre, présenté au National Theatre puis en tournée, fut programmé sur BBC2. Voir aussi la série «Renaissance Classics» et «Renaissance Theatre» (intitulée «a night at the theatre»), par exemple le Twelfth Night de Branagh, passé de la scène à la télévision et maintenant disponible en vidéo. D'autres mises en scène précédemment rôdées au théâtre, comme le Macbeth de Trevor Nunn, ont été soumises à un re-formatage pour la télévision et enregistrées en studio. Voir à ce propos l'analyse de Michael Mullin qui étudie comment Trevor Nunn exploite les ressources télévisuelles pour retransmettre au plus près l'expérience théâtrale : «Stage and Screen: The Trevor Nunn Macbeth», Shakespeare Quarterly, $n^{\circ} 38$, Autumn 1987, p. 350-59; reproduit dans Shakespeare on Television, J. C. Bulman \& H. R. Coursen (eds), Hanover \& London, University Press of New England, 1988, p. 107-15. Trevor Nunn avait déjà filmé, en 1974, son Antony and Cleopatra, originellement mis en scène pour le grand théâtre de Stratford et il reprit aussi, en 1990, son Othello, mis en scène, comme Macbeth, pour The Other Place.

${ }^{19}$ De même que l'on peut redistribuer un film que l'on croyait perdu, comme l'othello d'Orson Welles.

20 «Théâtre et cinéma», Qu'est-ce que le cinéma?, p. 160.

${ }^{21}$ Ici encore l'intérêt pédagogique est évident. II est très facile de faire percevoir à des étudiants que, dans une perspective shakespearienne, le meurtre de Duncan filmé par Polanski est un impossible parce qu'il modifie le rapport du spectateur à Macbeth dans la scène dite du meurtre. 
22 Voir par exemple le jugement porté par le critique théâtral Michael Billington, sur la mise en scène récente de Measure for Measure de S. Braunschweig pour le Festival d'Édimbourg, ensuite présentée au Théâtre des Amandiers, à Nanterre: «it lacks the cinematic fluency we expect in modern Shakespeare. Rather than melt into each other, scenes are divided up by the onward march of the revolve» (The Guardian, 24 Aug. 1997). II est symplomatique que la critique vise essenticllement ce dispositif théâtral par excellence qu'est le plateau tournant. On peut aussi se demander s'il n'existe pas un lien entre la familiarité croissante, induite par la vidéo, avec l'interventionisme du réalisateur (en particulier dans les films d'auteurs comme ceux de Welles, d'Olivier et maintenant de Branagh) et la place prépondérante prise au théâtre par le metteur en scène. 